\title{
Synthesis and Resist Properties of Calixarene Polymers with Pendant Haloalkyl Groups
}

\author{
Hiroto Kudo $^{1 *}$, Hiroki Ogawa ${ }^{1}$, Hiroki Yamamoto ${ }^{2}$, and Takahiro Kozawa ${ }^{2}$ \\ ${ }^{1}$ Department of Chemistry and Materials Engineering, Faculty of Chemistry, Materials \\ and Bioengineering, Kansai University, 3-3-35, Yamate-cho, Suita-shi, Osaka, 564-8680, \\ Japan \\ ${ }^{2}$ Institute of Scientific and Industrial Research, Osaka University, 8-1 Mihogaoka, \\ Ibaraki, Osaka 567-0047, Japan
}

Keywords: calixarene, botryosin, electron beam, extreme ultraviolet, resist

\section{Introduction}

Calixarenes (CA's) are cyclic oligomers with pendant many hydroxyl groups and are easily synthesized by the condensation reaction of phenols and aldehydes in satisfactory yields under dynamic covalent chemistry system [1]. The synthesis and property of various calixarenes such as $p$ - $t$-butylcalix[8]arene (BCA[8]), $p$-methylcalix[6]arene $(\mathrm{BCA}[6])$, $c$-methylcalix[4]resorcinarene (CRA[4]), and c-(4-hydorxybenz)calix[4]resorcinarene (CRA-OH[4]) have been reported [2,3]. Their derivatives with pendant functional groups can be also synthesized easily.

Furthermore, polymers bearing $\mathrm{CA}$ moieties in the main or side chains (CA-polymers) have also been synthesized and they have potential applications as chemical sensors, selective membranes, and catalysts, as well as in microelectronic devices [4-6]. Very recently, we considered the synthesis of three dimensional cross-linking materials including calixarene moieties and expected that higher selectivity with some metal ions might show in the comparison of CA's derivatives. Indeed, when we examined the polyaddition reaction of $\mathrm{BCA}[8]$ and 1,6-hexanediisocyanate (HDI) in the presence of $\mathrm{NEt}_{3}$ in NMP at $25{ }^{\circ} \mathrm{C}$ for $12 \mathrm{~h}$, no gel compound could be obtained and only soluble copolymer poly(BCA[8]-co-HDI) was obtained in quantitative yield. Analysis by ${ }^{1} \mathrm{H}$ NMR, IR, MALDI-TOF mass spectroscopy, and AFM indicated that poly(BCA[8]-co-HDI) consists of nanoscale-gel-shaped structures that contain fixed holes and cavities resembling a bunch of grapes

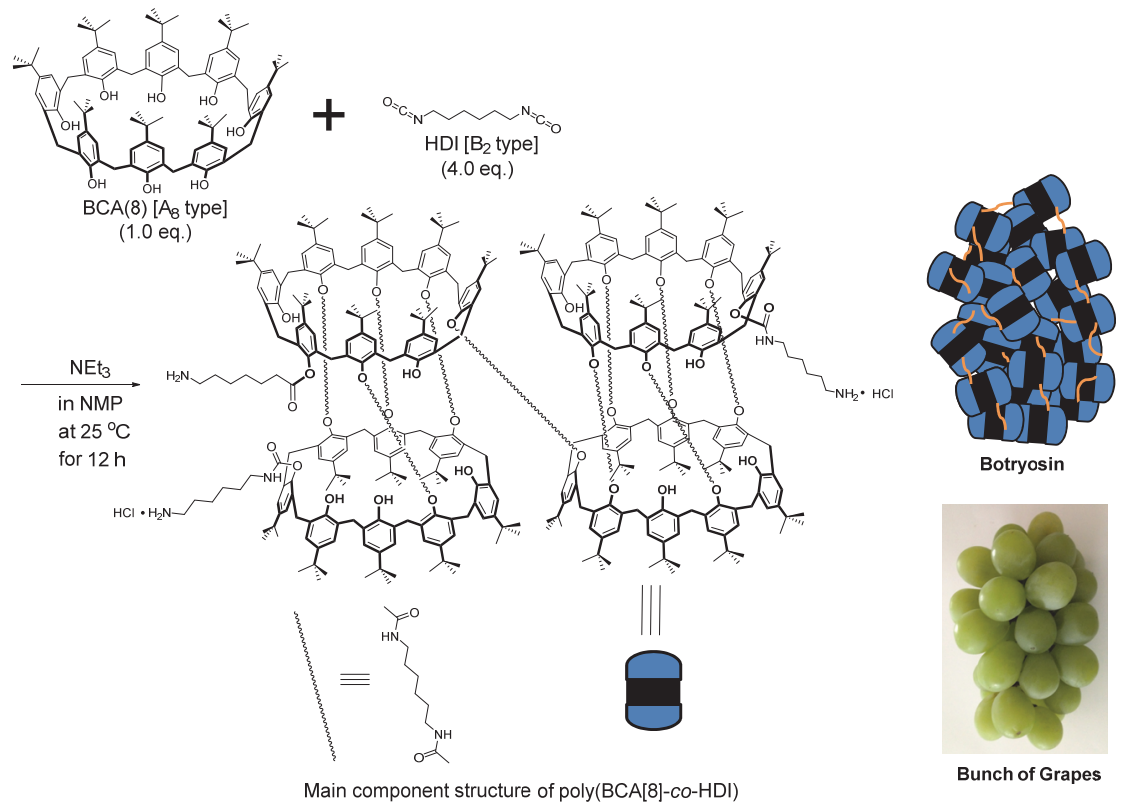

Scheme 1. Synthesis of botryosin-type polymer by the polyaddition of BCA[8] and HDI. 
(Scheme 1). We designated this class of polymers as botryosin, after the Greek word botrys, meaning a bunch of grapes [7].

In this time, we examined the condensation reaction of $\mathrm{BCA}[8]$ and adamantan derivative (AD) containing carboxylic acid groups, anticipating that soluble polymers have botryosin-like structure and ester groups in the main chain can be decomposed, to offer higher resolution resist pattern. The synthesis and resist properties of the polymers by the polycondensation of $\mathrm{BCA}[8]$ as a $\mathrm{A}_{8}$ type monomer with $\mathrm{AD}$ as a $\mathrm{B}_{2}$ type monomer.

\section{Experimental}

2.1. $p$-t-Butylcalix[8]arene (BCA[8]), $p$-t-Butylcalix[4]arene $\quad(\mathrm{BCA}[4]), \quad$ and C-(4- $t$-butylbenz)calix[4]resorcinarene (BCRA[4]) $\mathrm{BCA}[8]$ and $\mathrm{BCA}[4]$ were commercial available used. C-(4-t-butylbenz)calix[4]resorcinarene (BCRA[4]) was synthesized by the condensation reaction of resorcinnol and 4-t-butylbenz aldehyde using $\mathrm{HCl}$ as a catalyst in ethanol at $80{ }^{\circ} \mathrm{C}$ for $20 \mathrm{~min}$ in $51 \%$ yield.

\subsection{Synthesis of 1,3-Adamantane Dibromoacetate} (ADB)

Typical procedure: A solution of 1,3-adamantanediol $(2.00 \mathrm{~g}, \quad 11.9 \mathrm{mmol})$ 1,8-diazabicyclo[5.4.0] undec-7-ene (DBU) $(5.4 \mathrm{~g}$, $35.7 \mathrm{mmol})$ in NMP $(10 \mathrm{~mL})$ was prepared at $0{ }^{\circ} \mathrm{C}$. Then, bromoacetyl bromide $(7.2 \mathrm{~g}, 35.7 \mathrm{mmol})$ was added to the solution and stirred at $100{ }^{\circ} \mathrm{C}$ for $24 \mathrm{~h}$. After that $\mathrm{CHCl}_{3}(100 \mathrm{~mL})$ was added to the resulting mixture and then washed with $1 \mathrm{~N}$ hydrogen chloride solution, saturated $\mathrm{NaHCO}_{3}$ solution, and $\mathrm{NaCl}$ solution thrice. Organic solution was dried over anhydrous $\mathrm{MgSO}_{4}$ and was concentrated by evaporator. The obtained residue was purified by silica-gel column chromatography eluted with ethyl acetate/ hexane (volume ratio $1 / 1)$ to obtain white solid. Yield $=2.4 \mathrm{~g}(50 \%)$. $\mathrm{Mp}=57 \sim 61{ }^{\circ} \mathrm{C} . \quad$ IR $\left(\mathrm{KBr}, \mathrm{cm}^{-1}\right): 2921\left(\mathrm{v} \mathrm{CH}_{2}\right)$, 1745 (v C=O of ester), 1168 (v C-O of ether). ${ }^{1} \mathrm{H}$ NMR (400MHz, DMSO- $d_{6}$, TMS) $: \delta$ (ppm) 1.56 $\left(\mathrm{s}, 2 \mathrm{H},-\mathrm{CH}_{2-}\right) \quad 1.90 \sim 2.05\left(\mathrm{~m}, 8 \mathrm{H},-\mathrm{CH}_{2-}\right), 2.28$ $\sim 2.35$ (br, $2 \mathrm{H},>\mathrm{CH}-), \quad 2.44\left(\mathrm{~s}, 2 \mathrm{H},-\mathrm{CH}_{2}-\right), 4.26$ $\sim 4.30$ (m, $\left.4 \mathrm{H},-\mathrm{CH}_{2}-\mathrm{Br}\right)$.

2.3. Condensation Polymerization of $t$-Butylcalix[8]arene $(\mathrm{BCA}[8])$ and 1 , 3-Adamantane Dibromoacetate (ADB)
Typical procedure: A solution of $\mathrm{BCA}[8]$ (1.95 g, $1.5 \mathrm{mmol}$ ), tetrabutylammonium bromide (TBAB) $(1.51 \mathrm{~g}, 0.5 \mathrm{mmol})$, and $\mathrm{NaH}(0.288 \mathrm{~g}, 12$ mmol) in NMP (30 mL) was stirred at $80{ }^{\circ} \mathrm{C}$ for $3 \mathrm{~h}$. Then, ADB (2.45 g, $6.0 \mathrm{mmol})$ was added, and the reaction mixture was stirred at $80{ }^{\circ} \mathrm{C}$ for $48 \mathrm{~h}$. The resulting mixture was poured into a large amount of $1 \mathrm{~N}$ hydrogen chloride solution to precipitate a solid, which was collected by filtration on a membrane filter (Millipore LAWPO 4700 pore size $0.45 \mu \mathrm{m}$ ). The obtained solid was purified by silica-gel column chromatography eluted with $\mathrm{CHCl}_{3}$. The resulting residue was soluble in $\mathrm{CHCl}_{3}$ and poured into large amount of methanol to precipitate the solid. The molecular weights were estimated by SEC and their structure was confirmed by ${ }^{1} \mathrm{H}$ NMR spectroscopy. Yield $=0.68 \mathrm{~g}(19 \%) . M_{\mathrm{n}}=6,900\left(M_{\mathrm{w}} / M_{\mathrm{n}}=1.20\right) . \quad$ IR $\left(\mathrm{KBr}, \mathrm{cm}^{-1}\right): 3464(v-\mathrm{OH}), 2954\left(v-\mathrm{CH}_{2}-\right), 1735(v$ C-O-C), 1481 ( $v \mathrm{C}=\mathrm{C}$ of aromatic), 1187 ( $v \mathrm{C}-\mathrm{O}$ of aromatic). ${ }^{1} \mathrm{H}$ NMR (400 MHz, DMSO- $d_{6}$, TMS) $\delta$ $(\mathrm{ppm})=0.66 \sim 1.34\left(\mathrm{br} \mathrm{m},-\mathrm{CH}_{3}\right), 1.34 \sim 1.65(\mathrm{br}$ m, $-\mathrm{CH}_{2^{-}}$), $1.82 \sim 2.10$ (br m, $-\mathrm{CH}_{2^{-}}$), $2.10 \sim 2.35$ (br m, >CH-), $3.65 \sim 4.81$ (br m, $-\mathrm{O}-\mathrm{CH}_{2^{-}}$), $6.33 \sim$ 7.56 (br m, aromatic $\mathrm{H}$ ).

2.4. Condensation Polymerization of $p$ - $t$-Butylcalix[4]arene $(\mathrm{BCA}[4])$ and 1 , 3-Adamantane dibromoacetate (ADB)

The reaction of $\mathrm{BCA}[8]$ and $\mathrm{ADB}$ was carried out in the same way for the reaction of $\mathrm{BCA}[8]$ and ADB. Yield $=24 \% . \quad M_{\mathrm{n}}=1,400\left(M_{\mathrm{w}} / M_{\mathrm{n}}=1.34\right)$. IR $\left(\mathrm{KBr}, \mathrm{cm}^{-1}\right): 3472(v-\mathrm{OH}), 2953\left(v-\mathrm{CH}_{2}-\right)$, $1754(v \mathrm{C}=\mathrm{O}), 1480(v \mathrm{C}=\mathrm{C}$ of aromatic), $1183(v$ $\mathrm{C}-\mathrm{O}) .{ }^{1} \mathrm{H}$ NMR $\left(400 \mathrm{MHz}, \mathrm{DMSO}-d_{6}, \mathrm{TMS}\right) \delta$ $(\mathrm{ppm})=0.81 \sim 1.32\left(\right.$ br m, $\left.-\mathrm{CH}_{3}\right), 1.71 \sim 1.96(\mathrm{br}$ $\mathrm{m},-\mathrm{CH}_{2}-$ ), $3.01 \sim 3.37$ (br m, $-\mathrm{CH}_{2}-$ ), $4.09 \sim 4.48$ (br m, >CH-), $4.49 \sim 5.17$ (br m, $-\mathrm{O}-\mathrm{CH}_{2^{-}}$), $6.30 \sim$ 7.21 (br m, aromatic $\mathrm{H}$ ).

2.5. Condensation Polymerization of C-(4-t-Butylbenz)calix[4]resorcinarene (BCRA[4]) and 1, 3-Adamantane Dibromoacetate (ADB)

The reaction of $\mathrm{BCRA}[4]$ and $\mathrm{ADB}$ was carried out in the same way for the reaction of BCA [8] and ADB. Yield $=78 \% . \quad M_{\mathrm{n}}=4,740$ $\left(M_{\mathrm{w}} / M_{\mathrm{n}}=2.30\right) . \quad$ IR $\left(\mathrm{KBr}, \mathrm{cm}^{-1}\right): 3450(v-\mathrm{OH})$, $2958\left(v-\mathrm{CH}_{2}-\right), 1734(v \mathrm{C}=\mathrm{O}), 1496(v \mathrm{C}=\mathrm{C}$ of aromatic), 1199 ( $v$ C-O). ${ }^{1} \mathrm{H}$ NMR $(400 \mathrm{MHz}$, DMSO- $d_{6}$, TMS) $\delta(\mathrm{ppm})=0.80 \sim 1.41(\mathrm{br} \mathrm{m},-$ $\mathrm{CH}_{3}$ ), $1.38 \sim 2.64$ (br m, $-\mathrm{CH}_{2}$ ), $3.80 \sim 4.88$ (br m, $-\mathrm{CH}_{2}$-b and $>\mathrm{CH}-$ ), $5.95 \sim 7.61$ (br m, aromatic $\mathrm{H}$ ). 
2.6. Thickness Loss Property on the Silicon Wafer after Soaking in $2.38 \mathrm{wt} \%$ Tetramethylammonium Hydroxide (TMAH) aq. Solution

The solutions of poly(BCA[8]-co-ADB), poly(BCA[4]-co-ADB), and poly(BCRA[4]-co-ADB) in propylene glycol monomethyl ether acetate (PGMEA) were spin-coated on the silicon wafer to prepare corresponding thin films with about $50 \mathrm{~nm}$ thickness. The value of thickness of the thin films was determined by ellipsometry before and after soaking in TMAH aq. for $30 \mathrm{sec}$.

\subsection{Resist Sensitivity}

The resist solutions were filtered through a $0.20 \mu \mathrm{m}$ PTFE syringe filter prior to spin-coating on silicon wafers which were primed with hexamethyldisilazane (HMDS). Spin-coating was performed at $3000 \mathrm{rpm}$ for $30 \mathrm{~s}$ to form thin films on the silicon wafers. Then these spin-coated films were prebaked at $150{ }^{\circ} \mathrm{C}$ for $60 \mathrm{~s}$. The film thickness was adjusted to $100 \mathrm{~nm}$. The resulting films were exposed to EUV lithography (energetic EQ-10M) [8]. The area of exposure was approximately $1 \times 1 \mathrm{~cm}^{2}$. After the exposure, they were developed by dipping in THF at $23{ }^{\circ} \mathrm{C}$ for $30 \mathrm{~s}$ and then rinsed in deionized water before drying. The resist film thickness was measured with an ET200 surface profiler to obtain sensitivity curves.

\subsection{Etching Durability}

The etching rates of the thin films on the silicon wafers were determined using a reactive ion etching (RIE) plasma process. The plasma conditions were a $15 \mathrm{SCCM} \mathrm{CF}_{4}$ gas flow, a 5 sccm Ar gas flow, and an RF power of $100 \mathrm{~W}$. The pressure in the process chamber was $1.0 \mathrm{~Pa}$.

\subsection{EB Patterning Property}

The mixture of polymer and triphenylsulfonium perfluoro-l-butanesulfonate (TPS-PFBS) as a photo-acid generator (PAG) was dissolved in PGMEA, which feeds weight- $\%$ ratio was as follow.; polymer / TPS-PFBS / trioctylamine $=100 / 10 / 1.25$. The spin-coating thin films on the silicon wafers were soft baked (SB) at $170{ }^{\circ} \mathrm{C}$ under $\mathrm{N}_{2}$ gas flow. The films were exposed to a $75 \mathrm{kV}$ EB (ELIONIX, ELS-7700). After the exposure, the films were post exposure bake (PEB) at $170{ }^{\circ} \mathrm{C}$ for $60 \mathrm{~s}$. They were developed by dipping $\mathrm{THF}$ at $23{ }^{\circ} \mathrm{C}$ for $30 \mathrm{~s}$ and then rinsed in deionized water for $30 \mathrm{~s}$ before drying. Resist patterns were recorded with a Hitachi-hitec S-5500 SEM.

\section{Results and Discussion}

3.1. Synthesis of Adamantane Derivative Containing Bromoalkyl Groups

The reaction of 1,3-adamantanediol and bromoacetyl bromide was carried out in the presence of DBU in NMP at $100{ }^{\circ} \mathrm{C}$, to give a corresponding 1, 3-adamantane dibromoacetate (ADB) in $50 \%$ yield.

3.2. Polycondensation Reaction of $p$ - $t$-Butylcalix[8]arene (BCA[8]), $p$-t-Butylcalix[4]arene $\quad(\mathrm{BCA}[4]), \quad$ and C-(4-tert-butylbenz)calix[4]resorcinarene (BCRA[4]) with 1,3-Adamantane Dibromoacetate (ADB).

The reaction of $\mathrm{BCA}[8]$ and $\mathrm{ADB}$ was carried out in the presence of DBU in NMP at 80 ${ }^{\circ} \mathrm{C}$ for 48h, yielding a soluble polymer poly(BCA[8]-co-ADB) with $M_{\mathrm{n}}=6,900$ and $M_{\mathrm{w}} / M_{\mathrm{n}}=1.20$ in $19 \%$ yield (Scheme 2 ).

Table 1. Synthesis of poly(BCA[8]-co-ADB), poly(BCA[4]-co-ADB), and poly(BCRA[4]-co-ADB). ${ }^{\mathrm{a}}$

\begin{tabular}{|c|c|c|c|c|c|}
\hline Polymer & ${\text { Yield }(\%)^{\mathrm{b}}}$ & $M_{\mathrm{n}}\left(M_{\mathrm{w}} / M_{\mathrm{n}}\right)^{\mathrm{c}}$ & $\mathrm{DR}(\%)^{\mathrm{d}}$ & $\mathrm{T}_{\mathrm{d}}^{\mathrm{i}}\left({ }^{\circ} \mathrm{C}\right)^{\mathrm{e}}$ & $\begin{array}{c}\text { Film-forming } \\
\text { Ability }\end{array}$ \\
\hline poly(BCA[8]-co-ADB) & 19 & $6,900(1.20)$ & 90 & 270 & good \\
poly(BCA[4]-co-ADB) & 24 & $1,400(1.34)$ & 76 & 283 & good \\
poly(BCRA[4]-co-ADB) & 78 & $4,740(2.30)$ & 70 & 185 & good \\
\hline
\end{tabular}

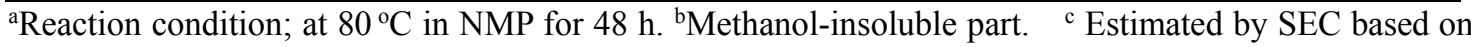
polystyrene standards; $\mathrm{LiBr}$ and phosphoric acid solution in DMF $(20 \mathrm{mM})$. ${ }^{\mathrm{d}} \mathrm{DR}$; degree of reaction ratio of hydroxyl groups calculated by ${ }^{1} \mathrm{H}$ NMR spectroscopy. ${ }^{\mathrm{e}} T_{\mathrm{d}}{ }^{\mathrm{i}}=$ Initial thermal decomposition temperature determined by TGA. 


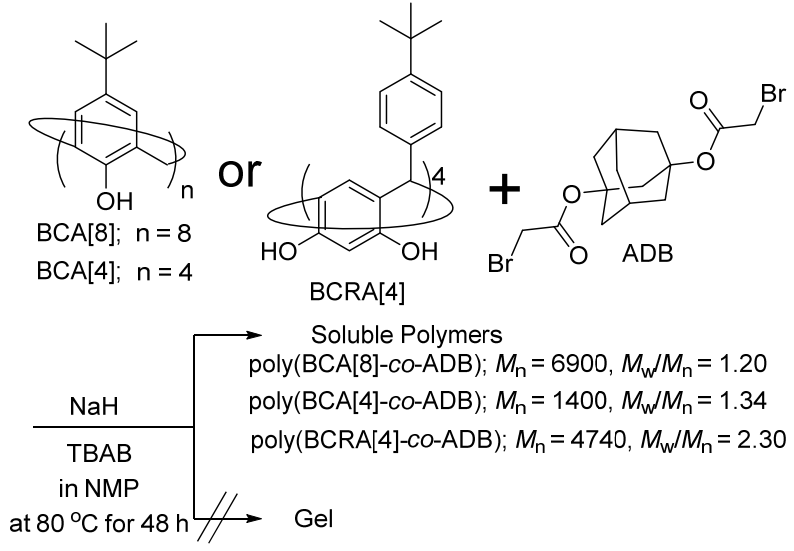

Scheme 2. Polycondensation reaction of $\mathrm{BCA}[\mathrm{n}](\mathrm{n}=$ 4 and 8) and BCRA[4] with ADB.

Its structure was confirmed by ${ }^{1} \mathrm{H}$ NMR and IR spectroscopy, which indicated that the polymer was consistent with both units of $\mathrm{BCA}[8]$ and ADB. The degree of reaction ratios (DR) was calculated by the decrease of integration ratios of the peaks assignable to hydroxyl groups at about $9.0 \mathrm{ppm}$ in ${ }^{1} \mathrm{H}$ NMR spectroscopy. This means that the polycondensation reaction $\mathrm{BCA}[8]$ and ADB proceeded not to afford gel but only soluble polymer. By the consideration based on our previous report, the soluble polymer might have botryosin-type structures as shown in Scheme 1 [7], presumably because that $\mathrm{BCA}[8]$ has fixed cone-shaped structures in which the hydroxyl groups are located at the lower rim and their polymerization proceeded homogeneously not to three dimensional cross-linked polymers. In the same way, the reaction of BCA[4] and BCRA[4] with ADB was performed to give the corresponding soluble polymers poly(BCA[4]-co-ADB) with $M_{\mathrm{n}}=1,400$ in $24 \%$ yield and poly(BCRA[4]-co-ADB) with $M_{\mathrm{n}}=$ 4,740 in $78 \%$ yield, respectively. These conditions and results are summarized in Table 1. Furthermore, thermal stability of the synthesized polymers was determined by thermo gravimetric analysis (TGA), and their initial decomposition temperatures $\left(\mathrm{T}_{\mathrm{d}}{ }^{\mathrm{i}}\right)$ were in the range between 184 and $270{ }^{\circ} \mathrm{C}$. These polymers were also soluble in common organic solvents such as DMSO, DMF, THF, $\mathrm{CHCl}_{3}$, and propyleneglycol monomethyl ether acetate (PGMEA). Their film-forming ability was confirmed by the spin-coating on silicon wafers from the polymers solutions in PGMEA, and it was observed that corresponding thin films with about $100 \mathrm{~nm}$ could be obtained from their solutions in PGMEA. These results indicate that poly(BCA[8]-co-ADB), poly(BCA[4]-co-ADB), and poly(BCRA[8]-co-ADB) are applicable to photoresist materials.

3.3. Thickness Loss Property on the Silicon Wafer after Soaking in TMAH aq.

Next, the value of thickness of the thin films prepared from the synthesized polymers was determined by ellipsometry before and after soaking in TMAH aq. for $30 \mathrm{sec}$, anticipating that these polymers are applicable to positive-type resist materials. These results are summarized in Table 2. In the case of the thin films of poly(BCA[8]-co-ADB) and poly(BCRA[4]-co-ADB), no decrease of their thicknesses were observed. However, $92 \%$-decrease was observed in the case of poly(BCA[4]-co-ADB). This means that poly $(\mathrm{BCA}[4]-c o-\mathrm{ADB})$ is inappropriate for resist material.

\subsection{Resist Sensitivity}

From the mentioned above results on thickness loss properties after soaking in TMAH aq., poly(BCA[8]-co-ADB) and poly(BCRA[4]-co-ADB) are applicable to positive type photo lithography system using TMAH aq. as a developer. Their resist-sensitivity was examined under EUV exposure tool. Solution of these polymers in the presence of $10 \mathrm{wt} \%$ of TPS-Nf as a PAG were spin coated on silicon wafer to prepare corresponding thin films in the absence of a quencher with about $100 \mathrm{~nm}$ thickness. The postexposure baking (PEB) temperatures and time were $150{ }^{\circ} \mathrm{C}$ and $60 \mathrm{~s}$, respectively. The value of the thickness of remained thin films was measured by means of ellipsometer after the lithography process. As the result, both thin films of poly(BCA[8]-co-ADB) and poly(BCRA[4]-co-ADB) were insoluble in

Table 2. The ratio of thickness lost after soaking in TMAH aq.

\begin{tabular}{|c|c|c|c|}
\hline Polymer & poly(BCA[8]-co-ADB) & poly(BCA[4]-co-ADB) & poly(BCRA[4]-co-ADB) \\
\hline Thickness Lost $^{\mathrm{a}}$ & $0 \%$ & $92 \%$ & $0 \%$ \\
\hline
\end{tabular}

aDetermined by ellipsometry. 
TMAH aq. solution fully after EUV exposure dose. This means that these polymers could not be applicable to positive type resist materials.

Next, we examined the possibility of that these polymers are applicable to negative-type resist materials. Solution of these polymers in the presence of $30 \mathrm{wt} \%$ of TPS-Nf as a PAG were prepared and their thin films were prepared in the same way as mentioned above. The value of the thickness of thin films was measured after EUV exposure dose using THF as a developer. Figure 1 depicts the sensitivity curve determined by the film-thickness on the silicon wafer.

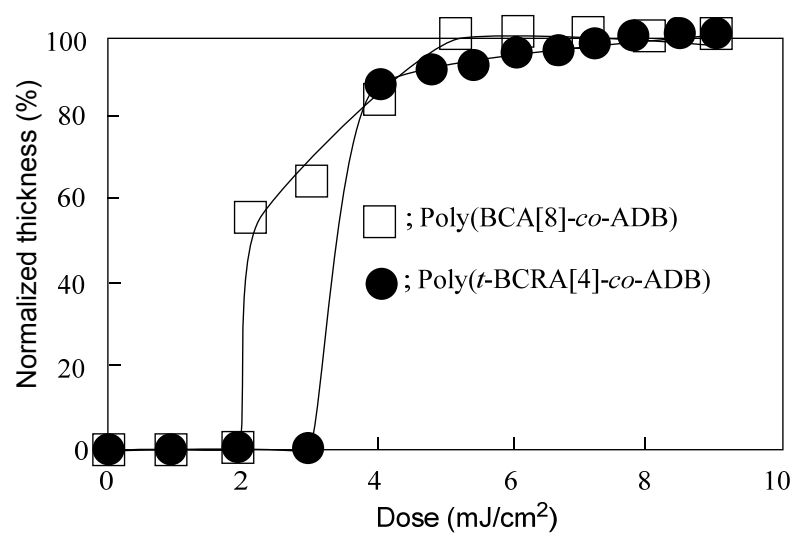

Figure 1. Resist-sensitivity curves of resist materials based on poly(BCA[8]-co-ADB) and poly(BCRA[4]-co-ADB).

Their thin-films were insoluble in THF after EUV exposure dose about $5 \mathrm{~mJ} / \mathrm{cm}^{2}$. This indicates that poly(BCA[8]-co-ADB) and poly(BCRA[4]-co-ADB) have adequate resist-sensitivity in the application of negative-type EUV photoresist system. Presumably because of that the synthesized polymers contained bromoalkyl groups in the side-chain, which might cause cross-linking reaction with EUV exposure to show negative-type sensitive curves.

\subsection{Etching Durability}

The etching durability was examined using RIE plasma process. The plasma conditions were a $15 \mathrm{sccm} \mathrm{CF}_{4}$ gas flow, a $5 \mathrm{sccm} \mathrm{Ar}$ gas flow, and RF power of $100 \mathrm{~W}$ under $1.0 \mathrm{~Pa}$ pressure in the process chamber. Figure 2 depicts the etching rate of the thin films prepared from poly(BCA[8]-co-ADB) and poly(BCRA[4]-co-ADB). Polymethyl methacrylate (PMMA) and polyhydroxy styrene (PHS) were also examined as typical polymers in the application of resist materials. It was observed that poly(BCA[8]-co-ADB) and poly(BCRA[4]-co-ADB) showed good etching durability.

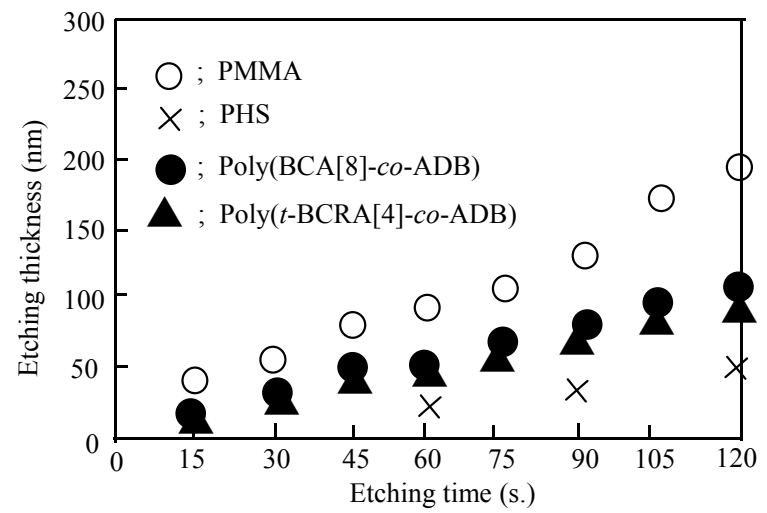

Figure 2. Etching durability of the thin films on the silicon wafer prepared from poly(BCA[8]-co-ADB) and poly(BCRA[4]-co-ADB), poly(methyl methacrylate) (PMMA), and poly(hydroxystyrene) (PHS).

\subsection{EB Patterning Property}

From the mentioned above results on resist sensitivity, negative resist pattern can be obtained using poly(BCA[8]-co-ADB) and poly(BCRA[4]-co-ADB). Patterning properties were examined using thin-film prepared from poly(BCRA[4]-co-ADB) in the EB exposure system. Figure 3 depicts SEM images of the resist pattern, which was obtained at $170{ }^{\circ} \mathrm{C}$ SB temperature and PEB temperature, and THF was used as a developer. A clear $100 \mathrm{~nm}$ resolution pattern with $83 \mathrm{~nm}$ of half-pitch was obtained using exposure dose at $640 \mu \mathrm{C} / \mathrm{cm}^{2}$.

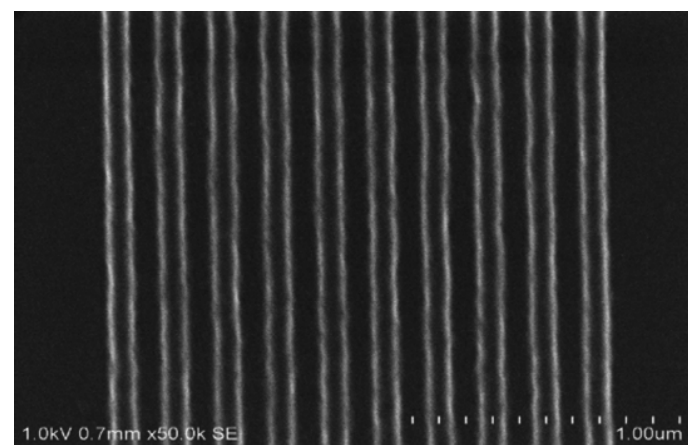

Line; $100 \mathrm{~nm}$, Half-Pitch; $83 \mathrm{~nm}$, Dose; $640 \mu \mathrm{C} / \mathrm{cm}^{2}$

Figure 3. EB-resist patterns obtained with poly(BCRA[4]-co-ADB) at exposure doses at 640 $\mu \mathrm{C} / \mathrm{cm}^{2}$.

This means that the present poly(BCA[8]-co-ADB) and poly(BCRA[4]-co-ADB) would offer negative 
resist pattern using THF as a developer.

In summary, we examined the condensation polymerization of $t$-butylcalix[8]arene (BCA[8]), $p$-t-butylcalix[4]arene $\quad(\mathrm{BCA}[4]), \quad$ and C-(4-t-butylbenz)calix[4]resorcinarene

(BCRA[4]) with 1, 3-adamantane dibromoacetate (ADB), yielding soluble polymers poly(BCA[8]-co-ADB), poly(BCA[4]-co-ADB), and poly(BCRA[4]-co-ADB), respectively. These polymers had good solubility, good film forming ability, and good thermal stability. It was anticipated that these polymers were applicable to positive-type resist materials. However, by the examination on the resist sensitivity using EUV exposure system, these polymers were applicable to negative type resist materials using THF as a developer. Furthermore, a negative clear resist pattern with $100 \mathrm{~nm}$ resolution could be obtained by EB exposure system. These results indicated that poly(BCA[8]-co-ADB) and poly(BCRA[4]-co-ADB) have high potential to offer higher resolution negative pattern using EUV lithography system.

\section{Acknowledgement}

This work was partly supported by Cooperative Research Program "Network Joint Research Center for Materials and Devices".

\section{References}

1. C. D. Gutch, "Calixarenes" Royal Society of Chemistry, Cambridge (1989).

2. Z. Asfari, V. Bohmer, J. Harrowfield, "Calixarenes 2001" Kluwer Academic Publishers (2001).

3. A. Ikeda and S. Shinkai, Chem. Rev., 97 (1997) 1713.

4. D. J. Cram, Science, 219 (1983) 1177.

5. A. Jasat and J. C. Sherman, Chem. Rev., 99 (1999) 931.

6. For example: P. Timmerman, W. Verbonnm and D. N. Reinhoudt, Tetrahedron, 52 (1996) 2663.

7. H. Kudo, M. Shizuma, K. Kubo and T. Hayashi, Chem. Lett., 44 (2015) 1765.

8. S. F. Horne, M. M. Besen, D. K. Smith, P. A. Blackborow and R. D'Agostino, Proc. SPIE, 6151 (2006) 61510P. 